Case Report

\title{
Peri-Implantitis - Decontaminating and Regenerative Treatment Protocol.
}

\author{
Duarte, Fernando Manuel Pinto ${ }^{*}$, de Oliveira, Leonel Alves ${ }^{*}$ \\ 1 University College of London - London -England; Clitrofa - Trofa - Portugal.; fduarte@clitrofa.com \\ 2 Nucleus of Applied Research in Morphology and Immunology - University of Brasilia; \\ profleonel2@gmail.com \\ * Correspondence: fduarte@clitrofa.com; profleonel2@gmail.com
}

\begin{abstract}
Peri-implant diseases are defined as pathological inflammatory reactions in the tissue surrounding the osseointegrated implants. They are classified into two categories: mucositis defined as peri-implant soft tissue inflammation and peri-implantitis - bone loss in the peri-implant region. A clinical case of a 61-years-old woman with an implant in the 46 anatomical position and a 5 -year follow-up is presented. A probe depth of $5 \mathrm{~mm}$ and a bone defect length of $35 \%$ was observed. The Implacure ${ }^{\circledR}$ Protocol was applied, with the main objective of eliminating the biofilm present on the exposed implant surface. There are multiple approaches to treat peri-implant diseases. While non-surgical treatment is essential for mucositis control, the treatment of peri-implantitis surgical treatment should be considered. Regenerative bone reconstruction promotes bone repair in the defect area and reduces bleeding during probing. To achieve that, autologous fibrin combined with Cerasorb $M \circledR$ was used. The proposed approach in the exposed clinical case involves the application of Implacure ${ }^{\circledR}$ Protocol, whose combination of the physical decontamination technique, together with the use of chlorhexidine and orthophosphoric acid, added with the combination Piperacillin + Tazobactam together with hyaluronic acid, provide a base that allows to regenerate bone using platelet-rich fibrin with Cerasorb $\mathrm{M} \circledast$ and increase the survival time of the implant.
\end{abstract}

Keywords: Peri-implantitis, Implacure ${ }^{\circledR}$ Protocol, Platelet-rich Fibrin

\section{Introduction}

The appearance of dental implants meant a change in the paradigm of oral rehabilitation, being currently accepted as the most effective method for rehabilitation of the patient's masticatory and aesthetic functions.

Peri-implant diseases are classified into two categories: mucositis and peri-implantitis. The mucosa is clinically diagnosed by the appearance of inflammation in the soft tissue near the implant, with no signs of loss of support bone. It may be accompanied by erythema, bleeding, and sometimes suppuration. Some authors propose, as diagnostic criteria, the presence of bleeding in the probe $\geq 4 \mathrm{~mm}$ in the absence of bone loss. ${ }^{(1,2)}$ Mucositis is reversible through a conservative approach with mechanical debridement and chemical treatment with chlorhexidine. Peri-implantitis involves periimplant bone tissue in addition to the mucosa. It is diagnosed radiologically, due to the loss of bone supporting the implant, accompanied by bleeding and probing depth $\geq 5 \mathrm{~mm}$. ${ }^{(3)}$

\subsection{Histology and Microbiology}

The microbiology of peri-implantitis is more diverse than that of periodontitis, with lower levels of complex red species. Histologically, peri-implantitis is much more infiltrative near the alveolar crest and often does not have a protective layer of tissue over the bone as we normally see in 
periodontitis. But the most important thing for clinicians is that diseases do not respond to treatment in a similar way. $(4,5,6)$

\subsection{Risk Factors}

Among the most important risk factors are the lack of oral hygiene, a history of periodontitis and tobacco consumption. Other factors, such as the metabolic control of diabetes, alcohol consumption, genetic susceptibility, absence of keratinized mucosa, type of implant surface or occlusion can also increase the risk of peri-implantitis. $(3,6)$ Despite this high prevalence, treatment options have been poorly studied; a 2011 Cochrane review concluded that the evidence available on the treatment for peri-implantitis is of insufficient quality and quantity and more and better research will be needed. (7)

\subsection{Treatment Options}

Non-surgical treatment of peri-implantitis using laser or air abrasive systems has shown insufficient results. Studies that evaluated chemotherapy and mechanical debridement show minimal resolution. The attempt to use photodynamic therapy to treat peri-implantitis was also unsuccessful. We can therefore say that, non-surgical treatments are not able to stop the progression of the condition. $(8,9,10)$

The only treatment that shows efficacy in resolving peri-implantitis appears to be surgical. However, resection surgery is only partially effective; Leonhardt et al. (11) described the effective surgical and antimicrobial treatment in just over half of peri-implantitis lesions over a five-year period. Heitz-Mayfield and collaborators ${ }^{(12)}$ demonstrated that an antimicrobial protocol with access to the surgical flap was able to stop the progression of peri-implantitis in $90 \%$ of cases in the short term (one year), but probing bleeding persisted in almost $50 \%$ of those same cases. Although a surgical approach to resection seems to improve the results, it is the combination of surgical approach and regenerative procedures where there is the highest success rate. Schwarz et al. ${ }^{(13)}$ found that regenerative surgical treatment is effective over 2 years, resulting in the stagnation of peri-implant bone loss and in the reduction of bleeding on probing from $80 \%$ to $34 \%$.

Unfortunately, not all peri-implant situations are conducive to regeneration. In some of these cases, the defect will present itself as a complete loss of the surrounding bone walls, leaving regeneration as an unpredictable treatment option. Aljateeli and collaborators ${ }^{(14)}$ proposed a decision tree based on the defect's morphology. If the defect had enough walls (two or more), regeneration would be attempted, but if there were zero or one wall, an apical repositioning flap would be suggested with implantoplasty (removal of exposed implant grooves and regularization of the surface with rotating instruments). The theoretical benefit of implantoplasty would be the production of a surface that is less favorable to bacterial colonization and a form of surface mechanical decontamination of the surface. Charalampakis and collaborators ${ }^{(15)}$ evaluated the longevity and the incidence of multiple different treatments recurrence in peri-implant patologies; more than half of the cases evaluated relapsed and were not properly controlled. This means that peri-implantitis is not only difficult to treat, but treated cases must be carefully monitored, since recurrence is common.

In all the treatments described, one of the critical steps is always the decontamination of implant surface. The complex topographies of modern surfaces offer an excellent refuge for bacterial growth, however, there are several options for decontaminating them. Anti-infectious treatments aim to detoxify the implant surface, where the following stand out: chlorhexidine, tetracycline, metronidazole, citric acid, laser and photodynamic therapy. Mechanical debridement aims to completely remove the biofilm, using: titanium, plastic or steel curettes, saline wash, cotton gauze, air abrasion or implantation. Some clinicians choose a combination of treatments in an attempt to ensure that surface decontamination is most successful. 
Several studies have evaluated the effectiveness of different surface treatments. Schwarz and collaborators ${ }^{(16)}$ compared surface decontamination using laser (Er: YAG) versus plastic curettes with cotton balls soaked in saline and found no noticeable differences in the final result. Romeo and collaborators (17-19) demonstrated that implantoplasty improved the non-regenerative surgical result of peri-implantitis, reducing the probing depths from 5.5 to $3.6 \mathrm{~mm}$ and the average bleeding index from 2.3 to 0,5 .

Implant surgery provides decontamination of the implant surface, but there are four concerns: a) heat production, b) deposition of implant material in the surgical area, c) damage to the implant surface and d) weakening of the implant's internal structure. Heat production is easily controlled through effective irrigation; depositions do not appear to be associated with any adverse clinical events. Removing the micro and macro-rough surface of the implant is commonly considered desirable, reducing the surface roughness to discourage bacterial colonization. The ultimate concern of weakening the implant's internal structure is not often discussed because the amount of surface to be removed is minimal.

\subsection{Implacure ${ }^{\circledR}$ Protocol}

The Implacure® Protocol consists of the application of an antibiotic solution of piperacillin and tazobactam in the peri-implant pocket in two sessions, separated by 4-7 days as an initial procedure. The tip of the needle should be bent to imitate a periodontal probe, and carefully the liquid inserted in the pocket, similar to the periodontal probe. When it reaches the bottom of the bag, the solution should be injected until the bag is completely filled. Make sure that the entire surface of the infected implant is covered with the liquid.

Subsequently, the implant decontamination protocol is followed: full thickness flap is performed to obtain adequate access to the treatment area, if possible with removal of the implant-supported crown, if it is screwed on. Perform a complete curettage of the infected bone, using drill no1 (black ring) to clean the implant surface on the cervical part and drill no2 (green ring) to clean the most apical grooves of the implant surface. Place a sterile compress around the implant to protect the bone walls and surrounding soft tissue; moisten with saline to improve adherence. Apply the gel composed of $37 \%$ orthophosphoric acid and $2 \%$ chlorhexidine over the entire implant surface using the syringe and let the gel act for 2 minutes to facilitate the disintegration of the biofilm. After 2 minutes, remove the gel with a sterile cannula and wash the implant surface with saline irrigation for 10 seconds, then remove the remaining saline solution with a sterile cannula and remove the compress. The next step consists of rolling the implant with a sterile compress, impregnated with the sodium hyaluronatepiperacillin-tazobactam solution; wait 5 minutes and remove the compress. Mix the bone graft Cerasorb $\mathrm{M} \circledR$ with the sodium piperacillin-tazobactam hyaluronate solution in a sterile container, place the bone graft in the defect and cover the area with a collagen membrane previously soaked with the sodium piperacillin-tazobactam hyaluronate solution and suture.

\subsection{Bone Regeneration Protocol}

The Cerasorb $\mathrm{M} \circledast$ is a resorbable and pure phase tricalcium beta-phosphate ceramics for filling, binding and reconstruction of bone defects as well as for bone fusion in the entire skeletal system. Cerasorb $M \circledR$ is made of biocompatible synthetic ceramic material with a phase purity of $\geq 99 \%$.

In the Cerasorb $M ®$ presentation the granules have a polygonal shape and, due to the open intercommunicating multiporosity composed of micro, meso and macropores (about 65\%), the radiopacity is lower and absorption is effected faster. Over months, in contact with the vital bone, the material is resorbed by the body and simultaneously replaced by autologous bone tissue. 
As a synthetic and bioactive ceramic material, Cerasorb $M \circledR$ has excellent histocompatibility and has no local or systemic toxicity. Unlike materials of biological origin, Cerasorb $\mathrm{M}{ }^{\circledR}$ presents no risk of infection or allergic reaction.

\subsection{Fibrin System ${ }^{\circledR}$ Protocol}

The platelet-rich fibrin are therapeutic blood matrices obtained by selective centrifugation acting as an adjuvant in tissue repair. For fibrin matrices obtention six samples of autologous blood were collected in $10 \mathrm{~mL}$ pure glass dry tube (Montserrat, Brazil) and two blood samples in polystirene dry tube (Greiner Bio-One, Austria), both centrifuged in the Fibrin System ${ }^{\circledR}$ centrifuge (Ortoalresa, Spain) according to the methodological proposal of Oliveira et al, $2020(20,21)$ that uses the relative centrifugal force (RCF) of $200 \mathrm{xg}$ for 10 minutes, for obtained two physical forms of fibrin, polymeric or solid gel form and monomeric or temporary liquid phase form in only step of spin. The liquid phase was used to obtain sticky bone with Cerasorb $\mathrm{M} \circledast$. The autologous fibrin membranes were applied as a recover surgical site.

\section{Case History}

A 61-years-old female patient who attended to our consultation, reporting painful symptoms at the level of the implant in the 4.6 anatomical position. The implant had been placed about 5 years ago. Complementary diagnostic exam (periapical x-ray) was performed, which allowed to see a significant bone loss of about $35 \%$ of the implant length and a probing depth greater than $5 \mathrm{~mm}$. As treatment plan it was proposed to perform a surgical approach treatment combining the protocols: Implacure ${ }^{\circledR}$, Fibrin System ${ }^{\circledR}$ and Peri-implant Bone Regeneration combined with Cerasorb $M{ }^{\circledR}$ (Curasan $\left.{ }^{\circledR}\right)$. The patient underwent systemic antibiotic, analgesic and anti-inflammatory therapy for 8 days. After 12 months of follow-up, the patient has a favorable clinical aspect, and a good recovery of bone trabeculation is visible radiologically. The patient has no symptoms. Figure 1. 


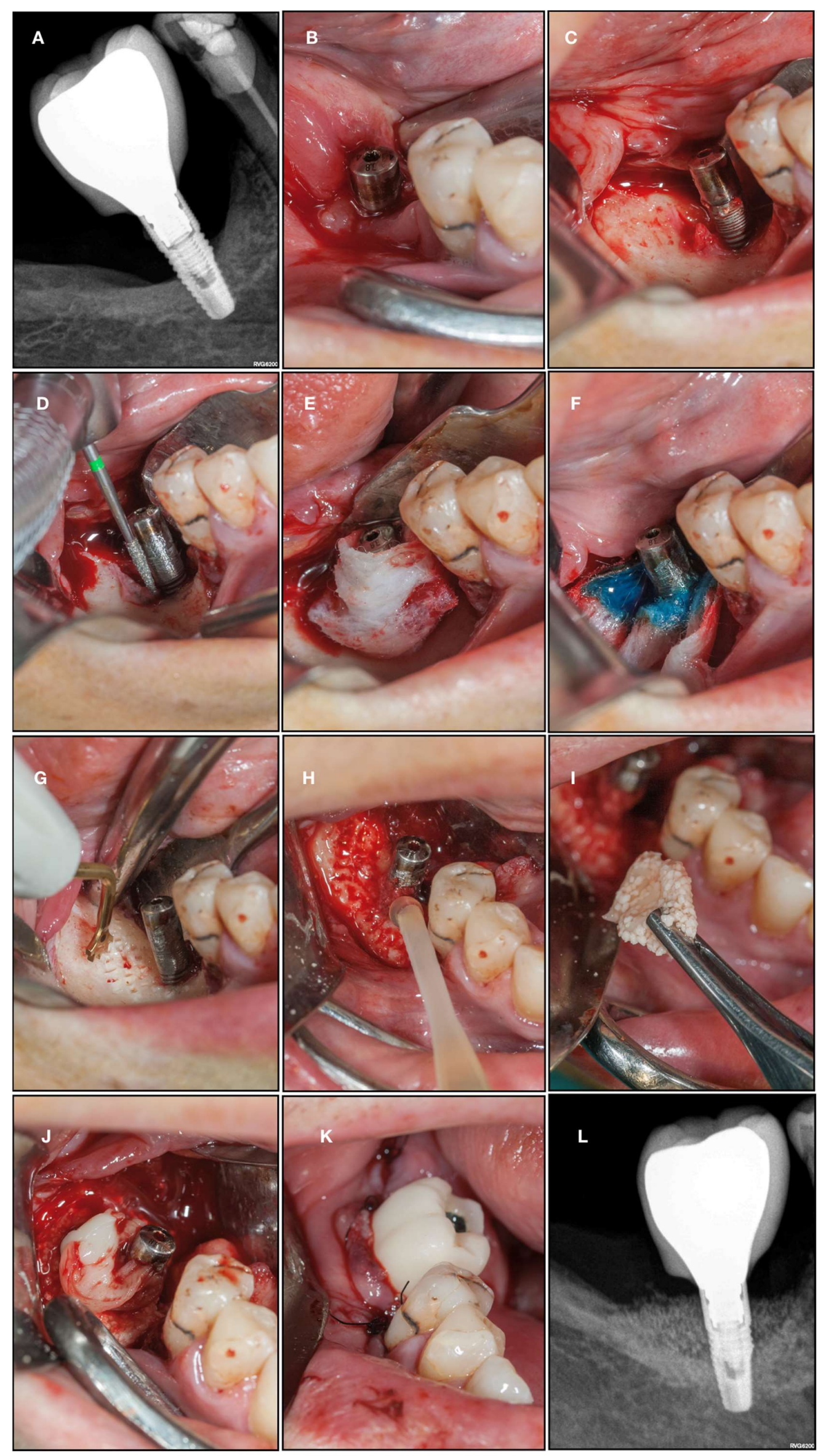


Figure 1. The sequence of image shows $(\boldsymbol{A})$ bone loss on initial radiography; $(\boldsymbol{B})$ removal of the crown and installation of a healing device to protect the prosthetic connection zone of the implant; $(\boldsymbol{C})$ surgical flap showing bone loss and exposure implant cervical zone; $(\boldsymbol{D})$ exposed surface remove with diamond cutting; $(\boldsymbol{E})$ bone bed protection; (F) orthophosphoric acid-H3PO4; $(G)$ piezosurgery decorticalization reaching the medullary bone; (H - I) monomeric phase fibrin dripping to generate a means of continuity and adhesion with the sticky bone; (J) polymeric fibrin membranes covering the graft material; $(\boldsymbol{K})$ temporary crown; $(\boldsymbol{L})$ radiograph control with 12-month follow-up.

\section{Discussion}

Peri-implant mucositis affects $80 \%$ of patients and $50 \%$ of implants, while peri-implantitis affects between $28-56 \%$ of patients and $12-43 \%$ of implants ${ }^{20)}$. Both pathologies have an infectious nature as a result of the accumulation of a biofilm. The main objective of treating peri-implantitis is to eliminate the biofilm from the implant surface; in this way the progression of the infection is stagnant and the function of the implant is maintained.

Resective surgery consists of removing soft tissues and the peri-implant pocket; and may be accompanied by implantoplasty, which consists of polishing the implant surface with a diamond drill. It has been shown that this procedure favors better results than if only soft tissue resection were performed. However, this treatment is discouraged in areas of aesthetic impairment, as it does not present favorable aesthetic results. Some studies have shown that the resective treatment can recover an average of $1.9 \mathrm{~mm}$ of bone in the area of the defect. In addition, by promoting biofilm control in the peri-implant area, the long-term stability of soft and hard tissues is favored. ${ }^{(18)}$

Regenerative surgery requires prior removal of granulation tissue from the peri-implant pocket and decontamination of the implant surface. In the literature, several antiseptics are proposed, such as 0.12 or 0.2 chlorhexidine; $3 \%$ hydrogen peroxide, sodium hypochlorite or saline. The use of several antibiotics is also proposed: amoxicillin / metronidazole, tetracycline / doxycycline or ciprofloxacin. The use of diode laser or Erbyum-YAG laser is also proposed to achieve implant decontamination. (17) In the present clinical case, it was decided to treat peri-implant infection by intrasulcular irrigation of piperacillin / tazobactam $100 / 12.5 \mathrm{mg}$ seven days before the intervention, in addition to the intraoperative application of orthophosphoric acid combined with chlorhexidine and again the solution piperacillin / tazobactam.

Once the implant surface has been decontaminated, there are numerous materials that can be used for the regeneration of the defect: xenografts, autografts, allografts, fluoridiazapatites and titanium granules. Studies have not shown the effectiveness of any of these regeneration materials over others. (18) In the present clinical case, the granules of the synthetic hydroxyapatite (Cerasorb $\mathrm{M}($ ) hydrated with a solution of piperacillin / tazobactam was mixed with liquid phase platelet-rich fibrin (20). The objective was to promote the initial stability and the continuous release of antibiotics for the biomaterial and the environment. ${ }^{(19)}$

The use of autologous fibrin in monomeric phase, in addition to serving as a binder to stabilize Cerasorb particles in the form of sticky bone, acted as a means of continuity between the medullary bone and the disinfected surface of the implant. The three-dimensional arrangement of the fibrin matrix favors cell transit allowing the progenitor cells from the bone marrow to be integrated into the graft set, constituting the tripod of tissue engineering. $(20,21)$

In the clinical case presented, there was a $4 \mathrm{~mm}$ reduction in the probing depth and no bleeding after probing.

It is essential to establish a maintenance protocol for the implants through frequent check-ups and a non-surgical approach to prevent the appearance of peri-implantitis. Once peri-implantitis is 
established, non-surgical treatment is not effective. The type of defect must be correctly diagnosed in order to choose the appropriate surgical protocol for each clinical case.

Mechanical and chemical decontamination seem to play an extremely important role in preventing the progression of this pathology.

Regenerative treatment promotes bone recovery in the defect area and reduces periodontal bleeding on probing.

The approach proposed in the exposed clinical case presupposes a combined treatment of implantoplasty, chemical decontamination and regenerative treatment that showed better results when compared with those in the literature; however, it is necessary to conduct multi-centric clinical studies with more significant samples and longer follow-up periods.

Conflicting Interest: The authors declare no conflict of interest.

\section{References}

1. Lindhe J, Meyle J. Peri-implant diseases: Consensus Report of the Sixth European Workshop on Periodontology. J Clin Periodontol. 2008; 35 (Suppl. 8): 282-5.

2. Renvert S, Roos-Jansåker AM, Lindahl C, Renvert H, Rutger Persson G. Infection at titanium implants with or without a clinical diag- nosis of inflammation. Clin Oral Implants Res. 2007; 18 (4): 509-16.

3. Karoussis IK, Müller S, Salvi GE, Heitz-Mayfield LJ, Brägger U, Lang NP.Association between periodontal and peri-implant conditions: a 10-year prospective study. Clin Oral Implants Res. 2004; 15(1): 1-7.

4. Koyanagi T, Sakamoto M, Takeuchi Y, Maruyama N, Ohkuma M, Izumi Y. Comprehensive microbiological findings in periimplantitis and periodontitis. J Clin Periodontol 2013; 40 (3):218-226

5. Berglundh T, Zitzmann NU, Donati M. Are peri-implantitis lesions different from periodontitis lesions? J Clin Periodontol 2011; 38 (Suppl. 11): 188-202.

6. Lang NP, Berglundh $\mathrm{T}$ on Behalf of Working Group 4 of the Seventh European Workshop on Periodontology: Periimplant diseases: where are we now? - Consensus of the Seventh European Workshop on Periodontology. J Clin Periodontol 2011; 38 (Suppl. 11): 178-181.

7. Esposito M, Grusovin MG, Worthington HV. Treatment of peri-implantitis: what interventions are effective? A Cochrane systematic review. Eur J Oral Implantol 2012;5 Suppl:S21-41.

8. Renvert S, Roos-Jansaker AM, Claffey N. Non-surgical treatment of peri-implant mucositis and periimplantitis: a literature review. J Clin Periodontol 2008; 35 (Suppl.8): 305-315.

9. Renvert S, Lindahl C, Roos Jansaker AM, Persson GR. Treatment of peri-implantitis using Er:YAG laser or an air-abrasive device: a randomized clinical trial. J Clin Periodontol 2011; 38: 65-73.

10. Meyle, J. Mechanical, chemical and laser treatments of the implant surface in the presence of marginal bone loss around implants. Eur J Oral Implantol. 2012;5 Suppl:S71-81.

11. Leonhardt A. Five-year clinical, microbiological, and radiological outcome following treatment of periimplantitis in man. J Periodontol 2003; 74:1415-1422.

12. Heitz-Mayeld LJA, Salvi GE, Mombelli A, Faddy M, Lang NP. Anti-infective surgical therapy of periimplantitis. A 12-month prospective clinical study. Clin. Oral Impl. Res. 23, 2012; 205-210

13. Schwarz F, Sculean A, Bieling K, Ferrari D, Rothamel D, Becker J. Two-year clinical results following treatment of peri-implantitis lesions using a nanocristalline hydroxyapatite or a natural bone mineral in combination with a collagen membrane. J Clin Periodontol 2008; 35: 80-87.

14. Aljateeli $\mathrm{M}, \mathrm{Fu} \mathrm{JH}$, Wang HL. Managing Peri-Implant Bone Loss: Current Understanding. Clin Implant Dent Relat Res. 2012 May;14 Supple 1:e109-18.

15. Charalampakis G, Rabe P, Leonhardt A, Dahle'n G. A follow-up study of periimplantitis cases after treatment. J Clin Periodontol 2011; 38: 864-871.

16. Schwarz F, Sahm N, Iglhaut G, Becker J. Impact of the method of surface debridement and decontamination on the clinical outcome following combined surgical therapy of peri-implantitis: a randomized controlled clinical study. J Clin Periodontol 2011; 38:276-284. 
17. Romeo E, Ghisolfi M, Murgolo N, Chiapasco M, Lops D, Vogel G. Therapy of peri-implantitis with resective surgery. A 3-year clinical trial on rough screw-shaped oral implants. Part I: clinical outcome. Clin Oral Implants Res 2005; 16:9-18.

18. Romeo E, Lops D, Chiapasco M, Ghisolfi M, Vogel G. Therapy of peri-implantitis with resective surgery. A 3-year clinical trial on rough screw-shaped oral implants. Part II: radiographic outcome. Clin Oral Implants Res 2007; 18:179-187.

19. Tomasi C, Derks J. Clinical research of peri-implant diseases quality of reporting, case definitions and methods to study incidence, pre- valence and risk factors of peri-implant diseases. J Clin Periodontol. 2012; 39 (Suppl. 12): 207-23.

20. de Oliveira LA, Borges TK, Soares RO, Buzzi M, Kückelhaus SAS (2020) Methodological variations affect the release of VEGF in vitro and fibrinolysis' time from platelet concentrates. PLoS ONE 15(10): e0240134. https://doi.org/10.1371/journal.pone.0240134

21. de Oliveira LA, Soares RO, Buzzi M, Mourão CFAB, Kawase T, Kuckelhaus SAS. Cell and platelet composition assays by flow cytometry: basis for new platelet-rich fibrin methodologies. J Biol Regul Homeost Agents. 2020 Jul-Aug,;34(4):1379-1390. doi: 10.23812/20-278-A. PMID: 32867466. 